\title{
A case of long-term observation of implants placed in the anterior region of a growing patient
}

\author{
Ju-Heon Lee', Min-Joong Kim¹, Pil-Young Yun ${ }^{1}$, Young-Kyun Kim,2 \\ ${ }^{1}$ Department of Oral and Maxillofacial Surgery, Section of Dentistry, Seoul National University Bundang Hospital, Seongnam, \\ ${ }^{2}$ Department of Dentistry \& Dental Research Institute, School of Dentistry, Seoul National University, Seoul, Korea
}

\begin{abstract}
This case report presents a long-term observation case after bone grafting and implant restoration in the anterior maxilla of an 18-year-old male patient. The patient underwent bone grafting and implant restoration in the maxillary central incisor region and was followed up for eight years. In the follow-up observation period two years after restoring the implant prosthesis, an occlusal discrepancy was observed, and it was decided that the prosthesis needed to be remade. An important point to consider in a single implant treatment is that the previously presented physiological age of maturity is not an absolute guideline for implant placement. Implants for maxillary incisors should be considered carefully in the growth phase and adults because the continuous eruption of adjacent teeth can cause esthetic issues even after the growth phase. (JOURNAL OF DENTAL IMPLANT RESEARCH 2021;40(3):80-84)
\end{abstract}

Key Words: Dental implant, Adolescent, Infraocclusion

\section{INTRODUCTION}

Since the development of implants by Branemark, implant placement is now being chosen as one of the general treatment options for tooth loss ${ }^{1}$. However, when implants are placed in a growing patient, several side effects can occur that have been thought as contraindications in principle. If permanent tooth loss occurs in a growing patient, a treatment method differing from that of an adult should be considered because of the patient's growth potential. Implant placement should only be mainly considered after growth is complete. Despite these principled contraindications, there are cases in which implants need to be placed early. In the early loss of permanent teeth due to trauma or surgical alveolar resection, and congenitally missing teeth from issues such as partial edentulous or ectodermal dysplasia, occlusal forces are not transmitted to the alveolar bone and thus the alveolar bone continues to be resorbed ${ }^{2,3)}$. In particular, if the ab- sence of maxillary incisors is over a long period of time, severe bone resorption of the buccal side is observed. This not only makes implant placement difficult, but also creates difficulty in achieving aesthetic satisfaction with the prosthesis. In addition, if maxillary incisors are lost at a young age, psychological factors such as inferiority due to aesthetic problems are also a factor in early implant placement ${ }^{3)}$.

In this study, we introduce a case of long-term observation after implant placement in a patient whose growth had not been completed, and present issues that occur when implants are placed in a growing patient. In addition, we would like to introduce considerations and guidelines for implant placement in growing patients.

IRB deliberation approved (IRB No. : B-2108/701-702).

\section{CASE REPORT}

On July 15, 2003, an 18-year-old male patient visited

Received July 20, 2021, Revised August 22, 2021, Accepted August 22, 2021 (c) Journal of Dental Implant Research.

This is an open access article distributed under the terms of the Creative Commons Attribution Non-Commercial License

(http://creativecommons.org/licenses/by-nc/4.0) which permits unrestricted non-commercial use, distribution, and reproduction in any medium, provided the original work is properly cited.

Correspondence to: Young-Kyun Kim, https://orcid.org/0000-0002-7268-3870

Department of Oral and Maxillofacial Surgery, Section of Dentistry, Seoul National University Bundang Hospital, 82 Gumi-ro 173, 173beon-gil, Bundang-gu, Seongnam 13620, Korea. Tel: +82-31-787-7541, Fax: +82-31-787-4068, E-mail: kyk0505@snubh.org 


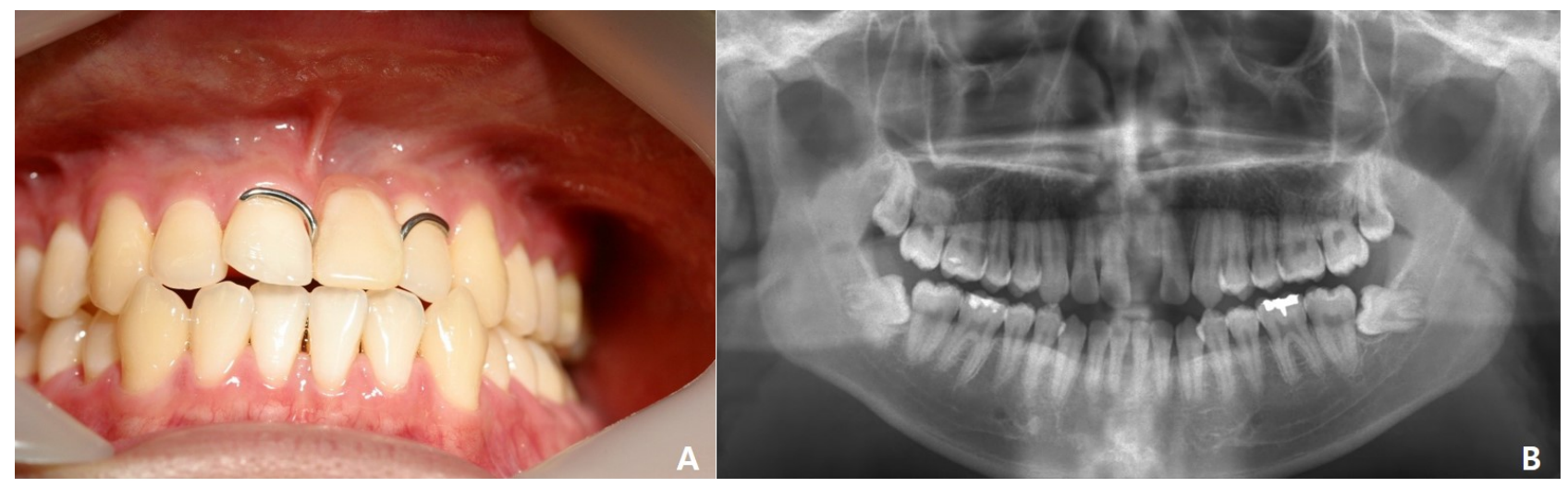

Fig. 1. (A) Flipper worn for 4 years before the initial visit. (B) Preoperative panoramic radiograph of the 18-year old male patient. \#21 tooth loss with severe bone atrophy.

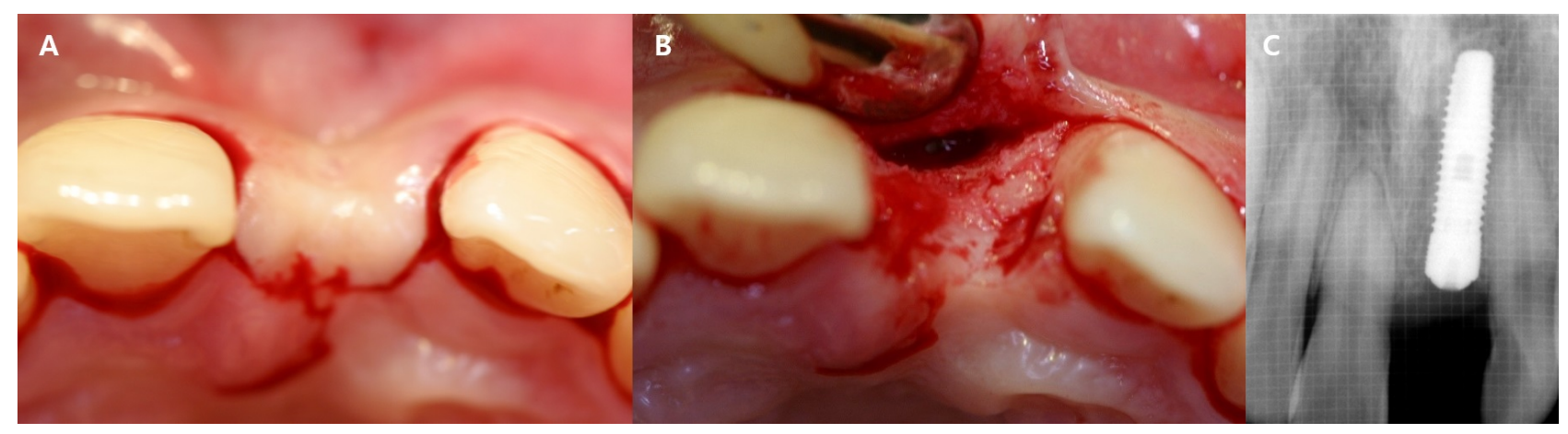

Fig. 2. (A) Palatal incision in order to decrease the risk of gingival recession and loss of interdental papilla. (B) Limited flap release to decrease the risk of labial bone loss. (C) Periapical radiograph after \#21 implant placement.

the hospital with a chief complaint of wishing to have an implant placed in the missing tooth area of \#21 (Fig. 1A). The patient had lost tooth \#21 4 years ago and was wearing a temporary flipper made by another dental clinic. The buccal-lingual width of the alveolar bone at the site of tooth loss was narrow (Fig. 1B). On August 12, 2003, the implant placement procedure was performed. Since the procedure was performed in an esthetic area, the incision line was created more palatally, and a limited flap was raised to the labial side. The implant (Implantium, Dentium, Seoul, Republic of Korea $4.5 \times 14 \mathrm{~mm}$ ) was placed while expanding the crestal bone using drills and osteotomes. A bone graft (DynaGraft ${ }^{\circledR}$, SeaSpine, Carlsbad, CA, USA) was performed in the labial subperiosteal pouch (Fig. 2). After surgery, the flipper was adjusted and fitted, and the healing period was good without any specific side effects. After 6 months of surgery, the second surgery was performed, the final prosthesis was PFG crown, and cemented using ZPC cement. The progress was observed while maintenance was performed at intervals of 6 12 months, and the \#21 implant prosthesis was found to be slightly intruded one year after the final prosthesis was placed (Fig. 3A). Afterwards, the patient did not visit the hospital for personal reasons, and on February 22, 2010, he visited and complained of discoloration of the implant prosthesis. Compared to previous findings, extrusive growth of the \#11 tooth had occurred and was now longer than the \#21 prosthesis (Fig. $3 \mathrm{~B}, 3 \mathrm{C})$. There was a plan to remake the prosthesis, but the patient never returned to the hospital and contact was lost (Fig. 3D).

\section{DISCUSSION}

When implants are placed in a growing patient, various issues may occur. During the growth period, the maxilla grows downward and anteriorly, while upward growth occurs in the mandible ${ }^{4,5}$. In particular, the ante- 


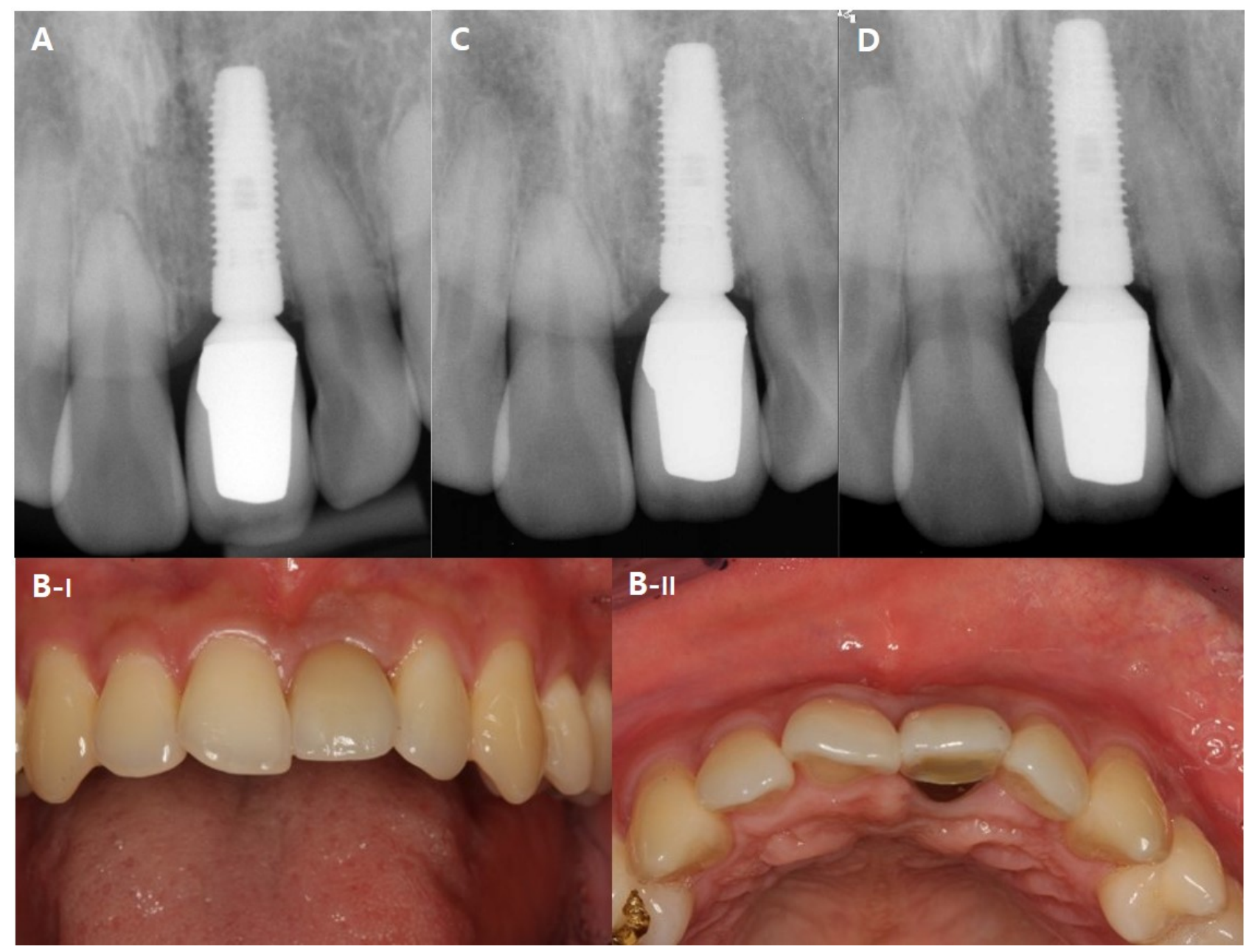

Fig. 3. (A) Postoperative periapical radiograph 1 year after final seating of the prosthesis. (B) Postoperative intraoral photograph 6 years after final seating of the prosthesis. (B-I) Labial view. (B-II) Occlusal view. (C) Postoperative periapical radiograph 6 years after final seating of the prosthesis. (D) Postoperative periapical radiograph 7.7 years after final seating of the prosthesis.

roposterior growth of the maxilla continues even after the age at which the lateral growth is completed. It is completed at the age of 14 or 15 for women and in their early 20 s for men ${ }^{6}$. And as the alveolar bone grows along with the eruption of the tooth, it grows vertically. If the surrounding alveolar bone grows in this way, and the implant is in a fixed position within the alveolar bone, the implant is gradually intruded ${ }^{7}$. When the implant is intruded into the bone, a deep pocket may be formed around the implant, and occlusal discrepancies may occur between the adjacent teeth and the implant prosthesis. In addition, since bone growth has not been completed in growing patients, there is often insufficient bone to place an implant, creating difficulties during placement. These issues are especially common in men and in maxillary central incisors, since males have a higher growth poten- tial than females, occlusal discrepancies from the adjacent teeth are more prominent compared to maxillary lateral incisors. When placing an implant in a growing patient, the most important thing to consider is the explanation of the side effects and obtaining patient consent. In particular, the possibility of remaking the prosthesis should be explained and the patient's consent for this possibility should be obtained. If implant placement is planned, it is recommended to delay implant placement until the age of 15 for women and 18 for men ${ }^{8)}$.

In this case, the age of the patient at the time of implant placement in the maxillary central incisor was 18 years old, which was at the boundary of the recommended physiological implant placement age. The reason the implant was placed immediately at the age of 18 was because the patient was sensitive to the esthetics and had 
a severe complex due to the loss of anterior teeth. When placing an implant in an esthetic area such as the maxillary anterior region, attention should be paid to various steps of the procedure such as the incision, flap elevation, and bone grafting. To minimize gingival recession and the loss of interdental papilla, the authors created a crestal incision that was biased to the palatal aspect with limited flap elevation to minimize labial bone resorption. In addition, to increase the volume of the labial bone and soft tissue, labial pouching was formed, and bone grafting was performed. However, although allogeneic bone has good osteoinductive healing, it is disadvantageous in maintaining labial volume with rapid resorption. In general, to maintain labial volume in the esthetic area, it is believed that it would be advantageous in terms of long-term esthetics to transplant xenogeneic or synthetic bone that exhibit slow or hardly resorbed resorption. It is believed that there are many issues in determining implant placement time in growing patients based on physiological age alone. In this case, occlusal discrepancies between the right central incisor and the implant prosthesis occurred during the follow-up period, which is thought to be due to the physiological eruption of the adjacent teeth and alveolar bone growth failure at the implant site, which occurred continuously after the growth period. Through the experience of this case, we learned that determining the time of surgery based on the physiological age of the completion of growth is not an absolute guideline for implant treatment in growing patients. Feher reported that the angle of the implant prosthesis changed over time at $8.6 \pm 1.3$ years after implant placement in the anterior maxilla of 21 adult patients ${ }^{9}$. As such, esthetic issues related to the continuous eruption of adjacent teeth or the growth of alveolar bone may occur even after the growth phase. Therefore, implant procedures should be performed with caution in esthetic areas such as the maxillary incisor region in growing patients as well as in adult patients. And there are more reliable standards to estimate growth cessation. It is known to be most reliable to superimpose serial cephalometric radiographs until no growth changes are seen for 1 year ${ }^{10)}$. However, this method is time consuming and may delay implant placement and cause tilting of adjacent teeth. Another method to estimate growth cessation is to evaluate skeletal growth using analysis of radiograph of hand and wrist ${ }^{11)}$. It even is advisable to determine growth cessation through a combination of several methods like evaluation of change in dental position and analysis of radiographs.

In principle, implant placement is contraindicated in growing patients, but if implants must be placed, esthetic side effects such as intrusion or height differences from adjacent teeth may occur due to alveolar bone growth and resorption. Therefore, it is necessary to create a careful treatment plan taking the growth into consideration, and to notify the patient in advance of the possibility of implant reoperation or remaking of the prosthesis. In general, considering growth, it is recommended that implant placement be delayed in women until the age of 15 and until the age of 18 for men, but this guideline should not be considered absolute and it should be recognized that each individual patient is different. Therefore, more reliable methods of evaluating bone growth should be used.

\section{ORCID}

Ju-Heon Lee, https://orcid.org/0000-0002-5213-0996

Min-Joong Kim, https://orcid.org/0000-0002-7387-1427

Pil-Young Yun, https://orcid.org/0000-0001-6097-1229

Young-Kyun Kim, https://orcid.org/0000-0002-7268-3870

\section{REFERENCES}

1. Adell R, Lekholm U, Rockler B, Branemark PI. A 15-year study of osseointegrated implants in the treatment of the edentulous jaw. Int J Oral Surg 1981;10:387-416.

2. Nemcovsky CE, Artzi Z, Moses O, Gelernter I. Healing of dehiscence defects at delayed-immediate implant sites primarily closed by a rotated palatal flap following extraction. Int J Oral Maxillofac Implants 2000;15:550-8.

3. Agarwal N, Kumar D, Anand A, Bahetwar SK. Dental implants in children: A multidisciplinary perspective for long-term success. Natl J Maxillofac Surg 2016;7:122-6.

4. Brahim JS. Dental implants in children. Oral Maxillofac Surg Clin North Am 2005;17:375-81.

5. Chen L, Liu J, Xu T, Lin J. Longitudinal study of relative growth rates of the maxilla and the mandible according to quantitative cervical vertebral maturation. Am J Orthod Dentofacial Orthop 2010;137:736 e1-8; discussion -7.

6. Bjork A, Skieller V. Growth of the maxilla in three dimensions as revealed radiographically by the implant method. $\mathrm{Br} J$ Orthod 1977;4:53-64. 
7. Kamatham R, Avisa P, Vinnakota DN, Nuvvula S. Adverse Effects of Implants in Children and Adolescents: A Systematic Review. J Clin Pediatr Dent 2019;43:69-77.

8. Mankani N, Chowdhary R, Patil BA, Nagaraj E, Madalli P. Osseointegrated dental implants in growing children: a literature review. J Oral Implantol 2014;40:627-31.

9. Feher B, Gruber R, Gahleitner A, Celar A, Necsea PL, Ulm C, et al. Angular changes in implants placed in the anterior maxillae of adults: a cephalometric pilot study. Clin Oral Investig 2021;25:1375-81.

10. Al-Taai N, Levring Jaghagen E, Persson M, Ransjo M, Westerlund A. A Superimposition-Based Cephalometric Method to Quantitate Craniofacial Changes. Int J Environ Res Public Health 2021;18.

11. Grave KC, Brown T. Skeletal ossification and the adolescent growth spurt. Am J Orthod 1976;69:611-9. 